\title{
On the method of lumens
}

\author{
Christopher A. Shera ${ }^{\text {a) }}$ \\ Eaton-Peabody Laboratories, Massachusetts Eye and Ear Infirmary, 243 Charles Street, Boston, \\ Massachusetts 02114
}

(Received 19 May 2014; revised 12 October 2014; accepted 22 October 2014)

\begin{abstract}
Parent and Allen [(2007). J. Acoust. Soc. Am. 122, 918-931] introduced the "method of lumens" to compute the plane-wave reflectance in a duct terminated with a nonuniform impedance. The method involves splitting the duct into multiple, fictitious subducts (lumens), solving for the reflectance in each subduct, and then combining the results. The method of lumens has considerable intuitive appeal and is easily implemented in the time domain. Previously applied only in a complex acoustical setting where proper evaluation is difficult (i.e., in a model of the ear canal and tympanic membrane), the method is tested here by using it to compute the reflectance from an area constriction in an infinite lossless duct considered in the long-wavelength limit. Neither the original formulation of the method-shown here to violate energy conservation except when the termination impedance is uniform-nor a reformulation consistent with basic physical constraints yields the correct solution to this textbook problem in acoustics. The results are generalized and the nature of the errors illuminated. (C) 2014 Acoustical Society of America.
\end{abstract}

[http://dx.doi.org/10.1121/1.4900919]

PACS number(s): 43.64.Bt, 43.64.Ha [KG]

Pages: $3126-3131$

\section{INTRODUCTION}

In papers principally devoted to modeling wave motion on the tympanic membrane, Parent and Allen (2007, 2010) introduced a novel and intuitive method for computing the plane-wave reflectance in a duct or tube (e.g., the ear canal) due to scattering at a boundary interface whose admittance varies across its surface (e.g., the eardrum). In this method, hereafter known as the "method of lumens," one imagines splitting the duct into multiple fictitious lumens-parallel, independent subducts-whose areas sum to the total area of the duct. (In anatomical usage, the word "lumen" refers to the inner cavity or bore of a hollow, tubular organ, such as a blood vessel.) At its closed end, each lumen sees only a small patch of the nonuniform boundary load. Although the load's acoustic properties vary from patch to patch, the number and shape of the lumens are chosen so that the admittance of each patch can be assumed constant, simplifying the acoustics of the lumen. After solving the reflection problem in each lumen separately, the results are combined across lumens to compute the plane-wave reflectance in the original duct. Although Parent and Allen $(2007,2010)$ emphasize the method's significant practical advantages (e.g., the ease with which it can be implemented in the time domain), and use the method to compute model responses for comparison with measured data, they provide no demonstration of its validity.

As recently discussed by Serwy (2014), Parent and Allen's (2007, 2010; hereafter known as P\&A) formulation of the lumen equations appears to violate relevant physical constraints, such as reciprocity and conservation of energy. Consequently, their formulation of the method cannot yield

\footnotetext{
a) Author to whom correspondence should be addressed. Electronic mail: christopher.shera@gmail.com
}

generally correct results. However, as Serwy (2014) further points out, P\&A's lumen equations can be repaired, and consistency with basic conservation laws restored, by implementing them using standard reciprocal scattering junctions (Bilbao, 2004, Sec. 1.1.3). The reader might therefore wonder whether the energy-corrected formulation of the method proves useful. Here, we assess the validity of both the original and revised formulations and determine the conditions under which they yield reliable results. Because the complex acoustics of the ear canal and tympanic membrane effectively preclude evaluation of the method in its original context, we test the method by applying it to a more tractable problem whose answer is well known. In particular, we use both the original (P\&A) and energy-corrected formulations to solve a textbook problem in acoustics-finding the lowfrequency, plane-wave reflectance in a duct with an area constriction. By analyzing the simplest test case possible, we exhibit the essentials of the method while eliminating numerical computations and the need for original source code, which has not been made publicly available.

\section{REFLECTANCE FROM AN AREA CONSTRICTION}

Consider the nonuniform, lossless duct shown in Fig. 1, in which the duct cross-sectional area decreases discontinuously from $A_{0}$ to $A_{1}$ at $x=0$. The abrupt area change causes partial reflection of incident waves. To test the method of lumens, we assume a plane wave incident from the left and compute the plane-wave reflectance, $R_{0}(x)$, at a distance $x$ from the constriction. By computing only the plane-wave reflectance, we ignore higher-order acoustic modes induced during the scattering process. When the wave frequency is sufficiently low (wavelength sufficiently long), these higherorder modes are evanescent and do not contribute to the 


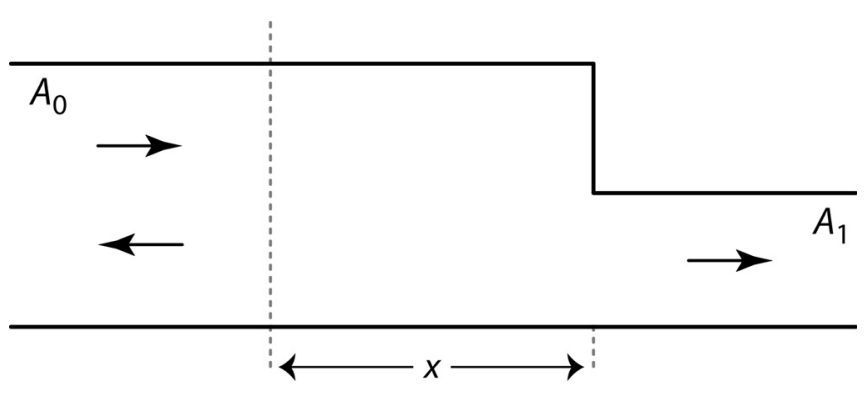

FIG. 1. Infinite acoustic duct with an area constriction. A plane wave incident from the left $(\rightarrow)$ is partially reflected $(\leftarrow)$ at the area change $(x=0)$. We compute the plane-wave reflectance in the plane of the vertical dotted line, located a distance $x$ from the discontinuity.

propagated pressure; the acoustics of the duct then remain one-dimensional except close to the discontinuity.

\section{A. Textbook method}

We first compute the reflectance using the traditional (but approximate) approach given in many acoustics textbooks (e.g., Blackstock 2000, Sec. 3.C; Kinsler and Frey 1962, Sec. 8.6). The single nonuniform duct with its area change is regarded as two uniform ducts joined at the discontinuity. The two semi-infinite ducts (duct 0 and duct 1 ) have characteristic admittances $Y_{0}=A_{0} / \rho c$ and $Y_{1}=A_{1} / \rho c$, respectively. By solving the one-dimensional wave equation in each of the two ducts and imposing continuity of pressure and volume velocity at the interface, one obtains

$$
R_{0}(x)=R_{0}(0) e^{-2 i k x},
$$

where

$$
R_{0}(0)=\frac{1-Y_{1} / Y_{0}}{1+Y_{1} / Y_{0}},
$$

and $k$ is the wave number. The phase shift $e^{-2 i k x}$ accounts for round-trip propagation between the plane of measurement $(x)$ and the discontinuity (0). Thus, the reflectance $R_{0}(x)$ is simply a phase-shifted version of the reflectance at $x=0$. If we let $A_{1} / A_{0}=\alpha$, then the reflectance $R_{0}(0)$ becomes

$$
R_{0}(0)=\frac{1-\alpha}{1+\alpha}
$$

For example, if $\alpha=\frac{1}{2}$, then $R_{0}(0)=\frac{1}{3}$. Equation (2) with $Y_{1}=A_{1} / \rho c$ is a low-frequency approximation applicable when the wavelength is much longer than any transverse dimension of the duct. Although the added precision proves unnecessary in the present context, one can improve the approximation and extend the region of validity by accounting for the inertial effects of non-planar modes induced by the discontinuity (e.g., Karal, 1953).

\section{Variation of the textbook method}

The textbook result can be recast in a form useful for comparing with the method of lumens. In the plane-wave limit, the area constriction at $x=0$ presents an acoustic load on duct 0 seen by the incident wave. To find the reflectance, one computes the total acoustic admittance of the load, which is found by integrating its specific admittance ( $\left.y_{\text {load }}\right)$ over the surface $(S)$ of the duct cross-section (area $A_{0}$ ). In Cartesian coordinates, for example,

$$
Y_{\text {load }}=\iint_{S} y_{\text {load }}(y, z) d y d z .
$$

The reflectance then becomes

$$
R_{0}(0)=\frac{1-Y_{\mathrm{load}} / Y_{0}}{1+Y_{\mathrm{load}} / Y_{0}}
$$

where $Y_{0}=A_{0} y_{0}=A_{0} / \rho c$.

In our example, the load on duct 0 consists of a "cap" of area $A_{0}$ consisting of two patches: A section of semi-infinite open duct (i.e., duct 1) with area $A_{1}=\alpha A_{0}$ and specific acoustic admittance $y_{1}=1 / \rho c=y_{0}$; and a section of hard wall with area $A_{0}-A_{1}=(1-\alpha) A_{0}$ and specific acoustic admittance $y_{\text {wall }}{ }^{1}$ The total acoustic admittance of the load thus becomes

$$
Y_{\text {load }}=\alpha A_{0} y_{1}+(1-\alpha) A_{0} y_{\text {wall }}=Y_{1}+Y_{\text {wall }} .
$$

Taking $y_{\text {wall }}=0$ for the hard wall gives $Y_{\text {load }}=Y_{1}$. Using this value of $Y_{\text {load }}$ in Eq. (5) reproduces the textbook result [cf. Eq. (2)].

For future reference, we consider the case when the cap on duct 0 consists of $N$ discrete patches of constant specific admittance. (In our simple example, $N=2$.) Indexed by $n$, each patch has area $A_{n}$, specific admittance $y_{n}$, and thus characteristic acoustic admittance $Y_{n}=A_{n} y_{n}$. Equation (4) becomes the simple summation, $Y_{\text {load }}=\sum_{n} Y_{n}$. Since the patch areas sum to the cross-sectional area of the duct $\left(\sum_{n} A_{n}=A_{0}\right)$, the ratio $Y_{\text {load }} / Y_{0}$ appearing in Eq. (5) for $R_{0}(0)$ can be written in the form

$$
Y_{\text {load }} / Y_{0}=\left(\frac{1}{A_{0}} \sum_{n=1}^{N} A_{n} y_{n}\right) / y_{0}=\left\langle y_{n}\right\rangle / y_{0},
$$

where $\left\langle y_{n}\right\rangle$ is the area-weighted average of the $y_{n}$.

To facilitate comparison with the method of lumens, we now express each admittance $y_{n}$ using an equivalent reflectance, $R_{n}$. The transformation from admittance $\left(y_{n}\right)$ to reflectance $\left(R_{n}\right)$, formally defined by

$$
y_{n} / y_{0}=\frac{1-R_{n}}{1+R_{n}},
$$

allows us to write the plane-wave reflectance $R_{0}(0)$ in terms of the individual patch reflectances, $R_{n}$. Tediously proved by algebra, the result for $N=2$ is

$$
R_{0}(0)=\frac{\alpha R_{1}+(1-\alpha) R_{2}+R_{1} R_{2}}{1+(1-\alpha) R_{1}+\alpha R_{2}} .
$$

As before, $R_{0}(x)$ is obtained by factoring in the round-trip phase shift in accordance with Eq. (1) [i.e., $R_{0}(x)=R_{0}(0) e^{-2 i k x}$ ]. Evaluating Eq. (9) using reflectances $R_{n}$ appropriate to the area constriction (i.e., $R_{1}=0$ and $R_{2}=1$ ) verifies that the formula reproduces the textbook result. 


\section{B. The method of lumens}

The method of lumens assumes, in effect, that the formally defined patch reflectances $R_{n}$ introduced in Eq. (8) characterize actual physical waves propagating in separate subcompartments of the duct. To compute $R_{0}(x)$ using the method of lumens, one therefore imagines splitting the terminal section of duct 0 into $N$ fictitious lumens of length $\ell$ (see Fig. 2 for $N=2$ ). The lumens have impenetrable but infinitely thin walls that isolate each lumen from the neighboring air space. By conservation of mass, the volume velocity of the incident plane wave partitions itself among the imaginary lumens in proportion to their areas, $A_{n}$, which sum to the total area of the duct. Each lumen has its own input reflectance, $\hat{R}_{n}$. The diacritical hat serves as a reminder that the $\hat{R}_{n}$ are defined at the lumen junction (i.e., at a distance $\ell$ from the termination) and therefore include roundtrip propagation delays. [The patch reflectances $R_{n}$ appearing in Eq. (8) are defined at the termination, located at $x=0$.]

\section{The P\&A formulation}

Although Parent and Allen $(2007,2010)$ provide no explicit formula for the reflectance, the equations defining their implementation of the method (the P\&A formulation) are encapsulated in their flow diagram (e.g., Fig. 4 of the 2007 paper and its reprise as Fig. 3 in 2010). Briefly, their computation proceeds as follows. Parent and Allen work with lumen wave volume velocities, $U_{n}^{ \pm}$. The superscripts indicate the direction of wave propagation: The $U_{n}^{+}$travel toward the tympanic membrane (i.e., the termination), the $U_{n}^{-}$away from it. Parent and Allen assume that the reflected volume velocity in the ear canal (i.e., in the main duct, with index 0 ) is the sum of the reflected volume velocities in the lumens $(n=1, \ldots, N)$. In other words, $U_{0}^{-}=\sum_{n} U_{n}^{-}$. (Unless otherwise indicated, all equations in this paragraph are understood to apply at the junction between the main duct and the entrance to the lumens.) The reflected volume velocities $U_{n}^{-}$can then be found from the $U_{n}^{+}$using the corresponding reflectances, $\hat{R}_{n}$; the summation therefore becomes $U_{0}^{-}=\sum_{n} \hat{R}_{n} U_{n}^{+}$. Since the total incident volume velocity $\left(U_{0}^{+}\right)$partitions itself among the lumens in proportion to their areas, one has $U_{n}^{+}=\left(A_{n} / A_{0}\right) U_{0}^{+}$. The P\&A formula for the reflectance at the lumen entrance becomes

$$
\hat{\mathcal{R}}_{0} \equiv U_{0}^{-} / U_{0}^{+}=\frac{1}{A_{0}} \sum_{n=1}^{N} A_{n} \hat{R}_{n}=\left\langle\hat{R}_{n}\right\rangle
$$

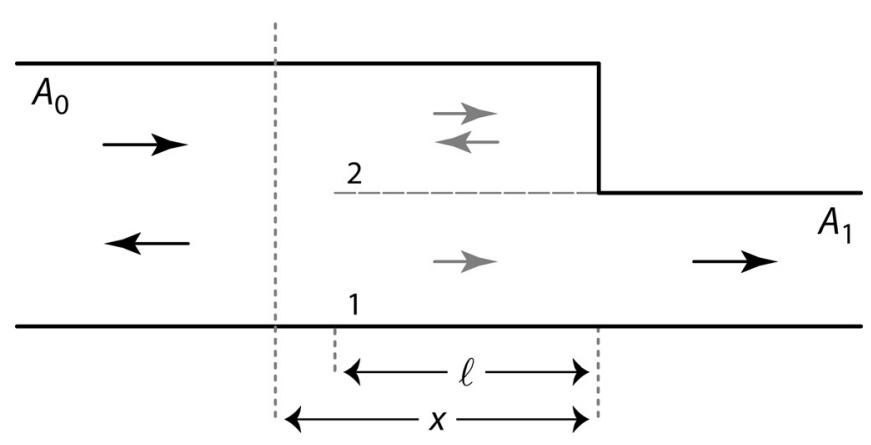

FIG. 2. The method of lumens splits the terminal section of duct 0 into imaginary subducts of length $\ell$, partitioning the volume velocity of the incident wave into separate channels (here denoted 1 and 2). The method assumes that wave propagation in each lumen is independent of the others. where the calligraphic font identifies the reflectance computed using the P\&A formulation and $\langle\cdot\rangle$ represents the areaweighted average of its argument. The reflectance at $x$ includes an additional phase shift due to propagation between $x$ and the lumen entrance: $\mathcal{R}_{0}(x)=\hat{\mathcal{R}}_{0} e^{-2 i k(x-\ell)}$. When the lumens are taken to align with the patches of constant $y_{n}$ defined above, then the reflectances $\hat{R}_{n}$ and $R_{n}$ are related by $\hat{R}_{n}=R_{n} e^{-2 i k \ell}$. The duct reflectance at $x$ therefore becomes

$$
\mathcal{R}_{0}(x)=\left\langle R_{n}\right\rangle e^{-2 i k x} .
$$

In the P\&A formulation, the duct reflectance $\mathcal{R}_{0}(x)$ is therefore proportional to the area-weighted average of the patch reflectances, $R_{n}$. [Contrast this with the textbook method summarized by Eqs. (5) and (7), which computes the reflectance using the area-weighted average of patch admittances.] For $N=2$, the P\&A formulation gives

$$
\mathcal{R}_{0}(x)=\left[\alpha R_{1}+(1-\alpha) R_{2}\right] e^{-2 i k x} .
$$

Note that Eq. (12) for $\mathcal{R}_{0}(x)$ differs from the value $R_{0}(x)$ derived using the textbook method [Eq. (9)]. Although the overall phase is correct, the magnitude is off. Indeed, evaluating the predicted reflectance magnitude using values appropriate for the area constriction (i.e., $R_{1}=0$ and $R_{2}=1$ ) gives $\left|\mathcal{R}_{0}\right|=|1-\alpha|$. Thus, for $\alpha=\frac{1}{2}$, the method of lumens predicts that $\left|\mathcal{R}_{0}\right|=\frac{1}{2}$, rather than the value $\left|R_{0}\right|=\frac{1}{3}$ given by the textbook method [see Eqs. (3) and (9)].

\section{Violation of energy conservation}

The discrepancy with the textbook result arises, in part, because P\&A's formulation of the lumen equations ignores basic physical constraints (Serwy, 2014). For example, one can show that the P\&A formulation violates energy conservation unless the termination is uniform. Energy conservation requires that the total power incident on the lumen junction equal the total power leaving it. When time-averaged over a cycle in the harmonic steady state, this condition becomes

$$
\underbrace{\left|U_{0}^{+}\right|^{2} / Y_{0}+\sum_{n=1}^{N}\left|U_{n}^{-}\right|^{2} / Y_{n}}_{\text {power incident on the junction }}=\underbrace{\left|U_{0}^{-}\right|^{2} / Y_{0}+\sum_{n=1}^{N}\left|U_{n}^{+}\right|^{2} / Y_{n}}_{\text {power leaving the junction }},
$$

where the $Y_{n}=A_{n} / \rho c$ are the real-valued characteristic acoustic admittances of the lumens (Bilbao, 2004, p. 15). Simplifying Eq. (13) using Parent and Allen's key assumptions $U_{n}^{+}=\left(A_{n} / A_{0}\right) U_{0}^{+}$and $U_{0}^{-} / U_{0}^{+}=\left(1 / A_{0}\right) \sum_{n} A_{n} \hat{R}_{n}$ [Eq. (10)], and using the identities $A_{0}=\sum_{n} A_{n}$ and $U_{n}^{-}=\hat{R}_{n} U_{n}^{+}$, yields an equation expressing the conservation of energy within the P\&A framework

$$
\left(\sum_{n=1}^{N} A_{n}\right)\left(\sum_{n=1}^{N} A_{n}\left|\hat{R}_{n}\right|^{2}\right)=\left|\sum_{n=1}^{N} A_{n} \hat{R}_{n}\right|^{2} .
$$

In general, Eq. (14) holds-that is, the P\&A junction conserves energy — only when the dependence on $\hat{R}_{n}$ factors from the sums and divides out; this requires either that $N=1$ or, equivalently, 
that the $\hat{R}_{n}$ be independent of $n$. In other words, energy conservation in the P\&A formulation requires that the termination be uniform, a trivial case that obviates any need for lumens. ${ }^{2}$ For example, using the values $\hat{R}_{1}=0$ and $\hat{R}_{2}=e^{-2 i k \ell}$ appropriate for the area constriction yields the condition $A_{0} A_{2}=A_{2}^{2}$ or, equivalently, $1-\alpha=(1-\alpha)^{2}$. This condition requires either $\alpha=0$ or $\alpha=1$. Thus, in our example involving an explicitly nonuniform termination $\left(\hat{R}_{1} \neq \hat{R}_{2}\right)$, the $\mathrm{P} \& \mathrm{~A}$ junction conserves energy only in the degenerate case that one of the two lumens has zero area so that the termination is de facto uniform.

\section{Energy-corrected formulation}

As explained by Serwy (2014), the violation of energy conservation in P\&A's implementation arises because of their misformulation of the junction equations that describe the lumen entrance. Although these equations conserve volume velocity, they do not conserve energy. Consistency with basic physical constraints at the lumen entrance can be restored by implementing the equations using reciprocal acoustic scattering junctions (Bilbao, 2004; Serwy, 2014). Does reformulating the equations in this way salvage the method of lumens?

Reformulating the lumen equations using reciprocal scattering junctions is equivalent to imposing continuity of pressure and volume velocity at each lumen entrance (e.g., Pierce, 1981, Sec. 7.3). Because these are exactly the same physical constraints imposed on the admittance patches at $x=0$ when deriving Eq. (9), one might guess that the energy-corrected method of lumens yields a formula for the reflectance at the lumen entrance identical to Eq. (9) but with the substitution $R_{n} \rightarrow \hat{R}_{n}$. Indeed, starting from the equations defining a standard scattering junction (Bilbao, 2004, Sec. 1.1.3), one can prove by algebra that for $N=2$,

$$
\hat{\mathcal{R}}_{0}^{\dagger}(\ell)=\frac{\alpha \hat{R}_{1}+(1-\alpha) \hat{R}_{2}+\hat{R}_{1} \hat{R}_{2}}{1+(1-\alpha) \hat{R}_{1}+\alpha \hat{R}_{2}},
$$

where the addition of the superscripted dagger ( $\dagger$ ) indicates use of the energy-corrected (physical) lumen equations. Rewriting Eq. (15) in terms of the $R_{n}$ and including the appropriate round-trip phase shifts yields

$$
\mathcal{R}_{0}^{\dagger}(x)=\frac{\alpha R_{1}+(1-\alpha) R_{2}+R_{1} R_{2} e^{-2 i k \ell}}{e^{2 i k \ell}+(1-\alpha) R_{1}+\alpha R_{2}} e^{-2 i k(x-\ell)} .
$$

Interestingly, Eq. (16) generally disagrees with the textbook result [Eq. (9)]. Taking the values $R_{1}=0$ and $R_{2}=1$ appropriate for the area constriction gives

$$
\mathcal{R}_{0}^{\dagger}(x)=\frac{1-\alpha}{1+\alpha e^{-2 i k \ell}} e^{-2 i k x},
$$

whose magnitude varies between $\frac{1}{3}$ and 1 , depending on $\ell$. Even when formulated in a manner consistent with basic physical constraints, the method of lumens evidently fails to predict the reflection from a simple area constriction.

\section{ABSTRACTION AND GENERALIZATION}

Although we simplified the analysis by considering an example involving the smallest non-trivial number of lumens
$(N=2)$, our conclusions are more general. Representing the three reflectance formulae compared here using an abstract "operator" notation provides a convenient means of comparing the various approaches while emphasizing the generality of the results. In the operator notation (explained below), the plane-wave reflectances have the respective values

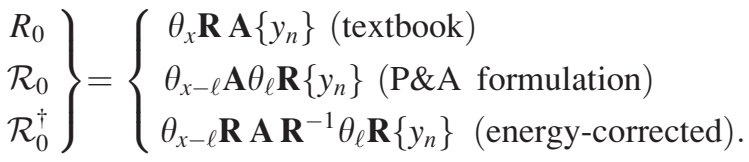

The operations (and the symbols representing them) include the following: Area-weighted averaging (A), transformation from the admittance to the reflectance domain $(\mathbf{R})$ and its inverse $\left(\mathbf{R}^{-1}\right)$, and phase shifting to account for round-trip wave propagation through a total distance $2 x\left(\theta_{x}\right)$. Note that the reflectance operator, $\mathbf{R}$, acts only on admittances, and the phase-shift operator, $\theta_{x}$, only on reflectances. In each case, the sequence of operations begins with the admittance patches $\left(y_{n}\right)$ that characterize the reflecting surface at $x=0$. The operators are then applied sequentially, from right to left. Thus, $\theta_{x} \mathbf{R A}\left\{y_{n}\right\}$ is shorthand for $\theta_{x}\left\{\mathbf{R}\left\{\mathbf{A}\left\{y_{n}\right\}\right\}\right\}$.

To translate Eq. (18) and its operator notation into words: The textbook computation of $R_{0}(x)$ involves (A) computing the area-weighted average of the $y_{n}$ to obtain $\left\langle y_{n}\right\rangle$ [Eq. (7)]; (R) transforming $\left\langle y_{n}\right\rangle$ to the reflectance domain to obtain $R_{0}(0)$ [Eq. (5)]; and $\left(\theta_{x}\right)$ phase-shifting $R_{0}(0)$ to obtain $R_{0}(x)$ [Eq. (1)]. Likewise, the P\&A formulation first finds the reflectance of each patch $(\mathbf{R})$, shifts its phase to the lumen entrance $\left(\theta_{\ell}\right)$, and then averages all $N$ phase-shifted reflectances (A) before finally propagating the result through the remaining distance out to $x\left(\theta_{x-\ell}\right){ }^{3}$ The energy-corrected formulation begins and ends similarly, but rather than averaging the shifted reflectances, transforms them into admittances $\left(\mathbf{R}^{-1}\right)$, averages those admittances $(\mathbf{A})$, and then transforms the average admittance back into a reflectance $(\mathbf{R})$. Since the operations in Eq. (18) do not, in general, commute with each other, the order in which they are applied matters. Thus, as we verified explicitly for $N=2$, the different approaches generally yield different results.

In addition to summarizing and generalizing our results, the operator representation is also helpful for identifying special circumstances when subsets of the three reflectance formulae agree with one another. For example, the $\mathrm{P} \& \mathrm{~A}$ formulation reproduces the textbook result, and all three equations reduce to the same sequence of operators, $\theta_{x} \mathbf{R}$, when the area-weighted averaging has no effect $(\mathbf{A}=\mathbf{1})$. This occurs when the termination is uniform; i.e., when there is only a single patch or, equivalently, when all $N$ patches have the same admittance, so that $y_{n}$ and $R_{n}$ become independent of $n .{ }^{4}$ As we prove in Sec. II B 2, the uniform termination is also the only case for which the P\&A formulation conserves energy at the junction. Since the uniform termination is easily solved by simpler means, the P\&A formulation evidently yields reliable results only when the introduction of fictitious lumens serves no computational purpose.

Another special case demonstrates the cyclic convergence of the energy-corrected method of lumens to the textbook 
procedure. According to Eq. (18) both reduce to the sequence $\theta_{x} \mathbf{R A}$, and therefore yield the same result, when $\theta_{\ell}=\mathbf{1}$. The lumen phase shift reduces to the identity when $e^{2 i k \ell}=1$; that is, when the lumen length $\ell=m \pi / k$, with $m=0,1, \ldots$. This condition is satisfied only when $\ell=0$ or when $\ell$ equals an integral number of half-wavelengths (or, equivalently, when the roundtrip delay within the lumens equals an integral number of periods of the stimulus frequency). For all other lumen lengths (or stimulus frequencies) the energy-corrected method of lumens gives erroneous results. Because the phase shift $e^{2 i k \ell}$ is periodic, the error introduced by the method varies periodically with $\ell$.

\section{DISCUSSION}

The method of lumens (Parent and Allen, 2007, 2010) was developed for the purpose of allowing easy, timedomain computation of the plane-wave reflectance in a duct terminated with a nonuniform acoustic load (e.g., the ear canal and tympanic membrane, respectively). At sufficiently low frequencies, a nonuniform load induces evanescent, non-planar modes that vary in the transverse direction but decay rapidly with distance from the load and do not contribute to the propagated plane-wave pressure. The acoustic field equations require that transverse pressure gradients (such as those occurring between adjacent lumens) induce corresponding flows that couple the system transversely. The method of lumens, however, assumes that the plane waves presumed to propagate within the fictitious lumens remain independent and non-interacting. (The lumens are presumed isolated from one another by thin, impenetrable walls.) Thus, the method of lumens ignores transverse coupling and cannot be used to find the pressure distribution within the section of the duct spanned by the imagined lumens. Despite this limitation, Parent and Allen tacitly assumed-but did not demonstrate-that the method of lumens accurately computes the plane-wave reflectance outside the luminal (evanescent) zone. To test this assumption, we applied the method to the simplest case we could construct: Finding the low-frequency, plane-wave reflectance from an area constriction in an infinite, lossless duct. The results demonstrate that neither the original P\&A formulation of the method nor a revision consistent with energy conservation yields the correct solution to this textbook problem in acoustics. ${ }^{5}$

In the P\&A formulation of the method, waves propagating in the different lumens remain completely uncoupled from one another. Were it not for the assumed independence of the lumens, variations in the reflectances $R_{n}$ from lumen to lumen would naturally give rise to transverse pressure gradients and induce the transverse flows necessary for wave spreading, for the evanescent decay of higher-order modes, and for the emergence of the plane-wave mode. When transverse coupling is prevented (e.g., by equations that assume the existence of impenetrable boundaries between the lumens), none of these things occur. Indeed, in the P\&A formulation, transverse pressure variations resulting from the nonuniform termination are preserved unchanged as the waves reflected within the lumens propagate back to the lumen entrance, where the reflected volume velocity in the main duct is found by summing the volume velocities in the individual lumens $\left(U_{0}^{-}=\sum_{n} U_{n}^{-}\right)$. As demonstrated in Sec. II B 2, this formulation violates energy conservation except when the duct termination is uniform and there are no transverse variations in pressure across the lumens.

Recasting the method using reciprocal scattering junctions (Bilbao, 2004, Sec. 1.1.3) restores consistency with basic physics but does not salvage the method. The energycorrected formulation couples the lumens together by requiring continuity of pressure and volume velocity at the lumen entrance, thereby ensuring the conservation of energy. Nevertheless, the energy-corrected formulation also fails to yield the textbook result for the reflectance due to an area constriction. The problem in a nutshell is that although the lumens are now physically coupled, they are coupled at the wrong place in the duct. In particular, they are coupled at the lumen entrance rather than near the constriction $(x=0)$, where the principal evanescent modes and transverse coupling occur in the actual duct. This difference in coupling location becomes important whenever the phase shifts (modulo $2 \pi$ ) due to round-trip wave propagation between the two locations become significant. Indeed, in Sec. III we saw that the energy-corrected formulation approaches the textbook result only as $\ell \rightarrow 0$ (or, more generally, as $\ell \rightarrow m \pi / k$, with $m=0,1, \ldots)$. For wideband stimuli, the energy-corrected method therefore yields textbook results only when the lumen phase shifts approach zero; that is, only in the limit $\ell$ $\rightarrow 0$ equivalent to the vanishing of the lumens. Thus, the discrepancy arises not because of any inherent physical inconsistency of the method, but because the lumen equations actually describe a physical system (namely, a duct with transverse coupling at the lumen entrance) completely different from the one they are assumed to represent (a duct with transverse coupling near $x=0$ ). Despite the method's intuitive appeal, the acoustics of a duct containing lumens, real or imagined, differs from those of a duct without.

Our results, together with those of Serwy (2014), indicate that Parent and Allen's $(2007,2010)$ representation of ear-canal acoustics using lumens is fundamentally flawed. Furthermore, their original formulation of the method cannot be "repaired" by implementing the lumens using reciprocal scattering junctions. Their model thus requires substantial revision before its ideas about wave propagation and reflection on the tympanic membrane can be accurately computed and compared with experiment.

\section{ACKNOWLEDGMENTS}

Supported by NIH grant R01 DC003687. The author thanks Jont Allen and Roger Serwy for useful discussions and Heidi Nakajima and Elizabeth Olson for helpful comments on the manuscript.

\footnotetext{
${ }^{1}$ Application of the method of lumens is most straightforward when the duct and the load have the same cross-sectional area. For this reason, we consider an area constriction, where part of the load can be considered a hard wall, rather than an area expansion.

${ }^{2}$ The statement that the termination is uniform when the $\hat{R}_{n}$ are independent of $n$ assumes that the round-trip phase shifts are identical across lumens. In Parent and Allen's $(2007,2010)$ application, the surface of the tympanic
} 
membrane (TM) is not perpendicular to the canal axis, and the distance to the TM from any location $x$ in the canal therefore varies across the TM. As a result, the round-trip phase shift depends on $n$. This raises additional complications not considered here.

${ }^{3}$ Because the averaging and phase-shifting operations commute, the P\&A formulation, represented in Eq. (18) as $\theta_{x-\ell} \mathbf{A} \theta_{\ell} \mathbf{R}\left\{y_{n}\right\}$, is equivalent to both $\theta_{x} \mathbf{A} \mathbf{R}\left\{y_{n}\right\}$ and $\mathbf{A} \theta_{x} \mathbf{R}\left\{y_{n}\right\}$.

${ }^{4}$ For $N=2$, it is easy to verify that when $R_{1}=R_{2}=R$, all three formulae give the same answer, namely, $R_{0}(x)=R e^{-2 i k x}$, independent of $\alpha$ [cf. Eqs. (9), (12), and (16)].

${ }^{5}$ As discussed in Sec. II A, the simple textbook value for the reflectance due to an area constriction is itself only a low-frequency approximation to the physically correct solution. The discrepancies noted here between the textbook value and results obtained using the method of lumens (either formulation) are of a qualitatively different kind than those implied by extensions such as the Karal correction-the method of lumens does not improve upon the textbook value; it merely degrades it.
Bilbao, S. (2004). Wave and Scattering Methods for Numerical Simulation (John Wiley and Sons, Chichester, UK), Sec. 1.1.3.

Blackstock, D. T. (2000). Fundamentals of Physical Acoustics (John Wiley and Sons, New York), Sec. 3.C.

Karal, F. C. (1953). "The analogous acoustical impedance for discontinuities and constrictions of circular cross section," J. Acoust. Soc. Am. 25, 327-334.

Kinsler, L. F., and Frey, A. R. (1962). Fundamentals of Acoustics (John Wiley and Sons, New York), Sec. 8.6.

Parent, P., and Allen, J. B. (2007). "Wave model of the cat tympanic membrane," J. Acoust. Soc. Am. 122, 918-931.

Parent, P., and Allen, J. B. (2010). "Time-domain 'wave' model of the human cat tympanic membrane," Hear. Res. 263, 152-167.

Pierce, A. D. (1981). Acoustics: An Introduction to its Physical Principles and Applications (McGraw-Hill, New York), Sec. 7.3.

Serwy, R. D. (2014). "Comment on 'Wave model of the cat tympanic membrane' [J. Acoust. Soc. Am. 122(2), 918-931 (2007)]," J. Acoust. Soc. Am. 135, 2493-2496. 